
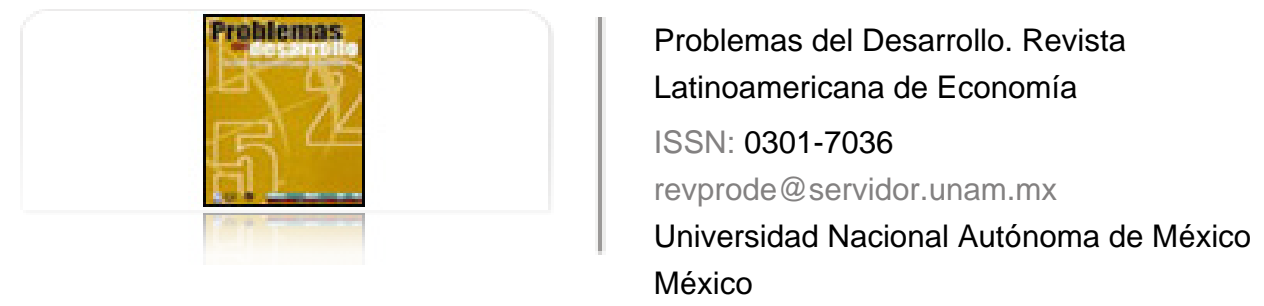

Mendoza Cota, Jorge Eduardo

IMPACTO DE LA INVERSIÓN EXTRANJERA DIRECTA EN EL CRECIMIENTO MANUFACTURERO

EN MÉXICO

Problemas del Desarrollo. Revista Latinoamericana de Economía, vol. 42, núm. 167, octubrediciembre, 2011, pp. 45-69

Universidad Nacional Autónoma de México

Distrito Federal, México

Disponible en: http://www.redalyc.org/articulo.oa?id=11820101003

- Cómo citar el artículo

- Número completo

- Más información del artículo

Página de la revista en redalyc.org

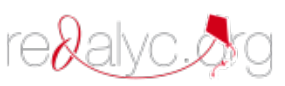

Sistema de Información Científica

Red de Revistas Científicas de América Latina, el Caribe, España y Portugal Proyecto académico sin fines de lucro, desarrollado bajo la iniciativa de acceso abierto 
Revista Problemas del Desarrollo, 167 (42), octubre-diciembre 2011

\title{
IMPACTO DE LA INVERSIÓN EXTRANJERA DIRECTA EN EL CRECIMIENTO MANUFACTURERO EN MÉXICO
}

\author{
Jorge Eduardo Mendoza Cota*
}

Fecha de recepción: 29 de noviembre de 2010. Fecha de aceptación: 28 de abril de 2011.

\section{RESUMEN}

El propósito de este artículo es aportar evidencia del impacto de la inversión extranjera directa (IED) en el crecimiento del sector manufacturero en el periodo 1999-2008. La metodología consiste en la estimación de un modelo econométrico de datos de panel al nivel de los 9 subsectores manufactureros de la economía mexicana. Los resultados muestran un efecto positivo de la apertura del sector manufacturero. Por otra parte, el efecto de la IED no es conclusivo estadísticamente. No obstante, cuando se considera en el modelo el crecimiento del personal calificado de la industria manufacturera puede considerarse a la IED como un determinante positivo para el crecimiento de este sector. Palabras clave: Inversión extranjera directa, sector manufacturero, modelo de datos de panel, economía mexicana, integración económica

\section{IMPACT OF FOREIGN DIRECT INVESTMENT ON THE GROWTH OF MANUFACTURING IN MEXICO}

\begin{abstract}
This article aims at providing evidence of the impact of foreign direct investment (FDI) on the growth of the manufacturing sector in the period 1999-2008. The methodology involves estimating an econometric model with panel data at the level of the nine manufacturing sub-sectors of the Mexican economy. The results show a positive effect from the opening of the manufacturing sector. Meanwhile, the effect of FDI is not statistically conclusive. However, when we include within the model the growth of skilled personnel in manufacturing industry, FDI can be considered as a positive determinant for growth in this sector.

Key words: Foreign direct investment, manufacturing sector, panel data model, Mexican economy, economic integration

* Investigador del Departamento de Estudios Económicos del Colegio de la Frontera Norte. Correo electrónico: emendoza@colef.mx
\end{abstract}


Jorge Eduardo Mendoza Cota

\section{L'IMPACT DE L'INVERSION ÉTRANGÈRE DIRECTE SUR LA CROISSANCE DE LA MANUFACTURE AU MEXIQUE \\ Résumé}

Le but de cet article est d'apporter la preuve de l'impact qu'ont eu les investissements étrangers directs (IED) sur la croissance du secteur manufacturier entre 1999 et 2008. La méthode employée consiste en une estimation du modèle économétrique de données de panel au niveau des 9 sous-secteurs manufacturiers de l'économie mexicaine. Les résultats montrent un effet positif de l'ouverture du secteur manufacturier. D'autre part, l'effet des IED n'est pas concluant statistiquement. Néanmoins, quand on considère la croissance dans ce modèle du personnel qualifié de l'industrie manufacturière, les IED peuvent être considérés comme un facteur positif pour la croissance de ce secteur. Mots clés : Investissements étrangers, secteur manufacturier, modèle de données de panel, économie mexicaine, intégration économique

\section{IMPACTO DO INVESTIMENTO ESTRANGEIRO DIRETO NO CRESCIMENTO MANUFATUREIRO NO MÉXICO \\ Resumo}

O propósito deste artigo é aportar evidencia do impacto do investimento estrangeiro direto (IED) no crescimento manufatureiro no período 1999-2008. A metodologia consiste na estimação de um modelo econométrico de dados de panel no nível dos 9 sub-setores manufatureiros da economia mexicana. Os resultados mostram um efeito positivo da abertura do setor manufatureiro. Por outra parte, o efeito do IED não é conclusivo estatisticamente. Não obstante, quando se considera no modelo o crescimento do pessoal qualificado da indústria manufatureira pode se considerar ao IED como um determinante positivo para o crescimento deste setor.

Palavras-chave: investimento estrangeiro direto, setor manufatureiro, modelo de dados de panel, economia mexicana, integração econômica.

\section{在製造業的外國直接投資在、墨西哥增長的影響}

摘要

本文的目的是提供外國直接投資在 1999 至 2008 年期間製 造業部門增長（FDI）的影響的證據。該方法涉及到面板 數據的計量經濟在製造業分 9 墨西哥經濟水平的估計。結 果顯示, 開放製造業產生積極影響。此外, 外國直接投資 的效果並不顯定論。然而，當在模型中考慮了外國直接投 資在製造業技能型人才的增長可以看作是對本部門增長的 積極因素

關鍵詞：外商直接投資，製造，面板數據模型，墨西哥的 經濟，經濟一體化 


\section{INTRODUCCIÓN}

Un componente de la estrategia de liberalización económica iniciada en la primera mitad de la década de los ochenta en México se relaciona con la política de promover la captación de la inversión extranjera directa (IED) en esa economía. Los resultados de esa estrategia han tenido éxito relativo debido a un importante crecimiento del mercado generado por este tipo de inversión (Ramírez, 2002). En este sentido, algunos autores han manifestado que en una economía de tamańo medio como la de México la IED puede tener un efecto de generar spillovers o derramamientos de tecnología (Love y Lage-Hidalgo, 2000). Por su parte, desde la perspectiva de la teoría del crecimiento endógeno, se ha generado evidencia de que el efecto de la IED es superior en economías que están abiertas y promocionan un crecimiento basado en las exportaciones (Balasubramanyam et al, 1996).

En este sentido, se ha señalado que la IED ha jugado un papel muy importante en el crecimiento de la economía mexicana debido al incremento de acervo de capital existente en la economía. Lo anterior ha implicado que el incremento de empresas con capital extranjero en la economía mexicana haya determinado por un lado, una mayor productividad del trabajo en las empresas que operan en México (Blomstrom y Persson, 1983). En particular, se ha argumentado que el incremento de la productividad está relacionado con el efecto de competencia que se genera entre empresas domésticas y de origen extranjero, particularmente en el sector manufacturero (Kokko, 1994).

Así pues, un aspecto relevante de la IED se relaciona con la presencia de la misma en el sector manufacturero lo que, de acuerdo con diferentes estudios (Aitken, Hanson y Harrison, 1997), (Blomström, Kokko y Zejan, 2000) (Jordaan, 2005), genera economías externas en dicho sector industrial, en especial en regiones donde existe alta concentración de IED.

Adicionalmente, existen diversos artículos sobre los determinantes de la localización y magnitud de la IED (Blomstrom y Persson, 1983), (Jordaan, 2008), (Dussel, Galindo, Loría y Mortimore, 2007), etcétera. Asimismo, se han realizado estudios para estimar el impacto de la IED en el crecimiento económico, como el trabajo de De Mello (1999) que analiza las economías de la oECD para el periodo 1979-1990, utilizando modelos de series de tiempo y de panel, para estimar la relación entre IED y la formación de capital. En lo correspondiente al efecto de la IED sobre el crecimiento económico, Borensztein, De Mello y Wha (1995) estimaron un modelo de panel que incluye 69 economías en desarrollo para el periodo 1970-1989. Los resultados muestran un efecto positivo aunque estadísticamente limitado de la IED en el crecimiento económico. 
Jorge Eduardo Mendoza Cota

Para el caso de México, Máttar, Moreno-Brid y W. Peres (2002) analizaron el efecto de las reformas económicas en la formación de capital y las tendencias e impacto de la IED en la industria manufacturera para el periodo 1982-2001. El estudio señala que existe poca evidencia de un impacto significativo de la IED en el crecimiento derivado de las reformas económicas de México. Por su parte, Ramírez (2000) realizó un análisis de cointegración sobre el efecto de la IED en productividad del trabajo para el periodo 1960-1995 encontrando que la IED, el capital privado y las exportaciones tienen un efecto positivo en la tasa de crecimiento de la productividad del trabajo.

Con respecto al efecto de la IED en el sector manufacturero se destaca el trabajo de Nunnenkamp, Alatorre y Waldkirch (2007), en el que se aplica un modelo de panel dinámico para analizar el efecto de la IED en el crecimiento del empleo de trabajadores del sector manufacturero mexicano en el periodo 19942006. Los resultados obtenidos apuntan a un impacto positivo, pero débil, de la IED en el crecimiento del empleo, particularmente de empleados calificados. Así pues, no existen muchos estudios conclusivos sobre el impacto de la IED en el crecimiento económico sectorial de México, particularmente sobre el sector manufacturero. Por ello, el propósito de este artículo es aportar estimaciones econométricas que permitan dilucidar el efecto de la IED en el crecimiento del sector manufacturero en México en la década del 2000. En ese sentido, el estudio se enfoca a realizar una estimación econométrica que permita adicionar evidencia estadística del efecto de la IED en el crecimiento del sector manufacturero de México al nivel de los 9 subsectores, para el periodo 2003-2007.

El artículo está dividido en las siguientes secciones. En la segunda sección se presenta un panorama de la evolución reciente de la IED orientada hacia el sector manufacturero al nivel de los principales subsectores y ramas. En la tercera parte se presenta y discute la construcción del modelo econométrico de panel que establece el crecimiento del sector manufacturero por subsectores como función de la IED entre otras variables. En la cuarta sección se presentan los resultados más relevantes de la estimación econométrica y, finalmente, en la quinta parte se presentan las conclusiones del trabajo.

\section{LA PARTICIPACIÓN DE LA INVERSIÓN EXTRANJERA DIRECTA EN LAS MANUFACTURAS DE MÉXICO}

Después de haber mostrado un dinámico crecimiento durante la década de los noventa, a partir del año 2000 se aprecia un declive de la participación de los flujos de la IED en el sector manufacturero de la economía mexicana en el total de 
captación de esos recursos financieros, cayendo un 55.3\% en ese año. Esta situación se deterioró aún más como resultado de la recesión económica de los EUA, y en el 2001 y el 2002 se experimentaron caídas de esa participación hasta llegar a un 19.8 y $37.1 \%$, respectivamente. Finalmente, se observa que después de un repunte en 2004, el porcentaje de la IED en el sector manufacturero volvió a caer en 2007 y 2008, además de presentarse este fenómeno en un contexto de una caída general de la IED total en México (Cuadro 1). Este último declive de los flujos de IED se dio como resultado del surgimiento de la crisis económica internacional de 2008. Finalmente, en lo relacionado al origen de la IED, se observa que los EUA siguen siendo la fuente más importante de esos flujos financieros, aunque es conveniente destacar que la proporción de la inversión extranjera directa de los EUA respecto al total de la IED captada en México se ha reducido considerablemente entre el 2001, en que presentó el mayor porcentaje respecto al total (71.2\%), y el 2008, año en que cayó su participación sensiblemente (45.7\%).

$\mathrm{Al}$ analizar la evolución de la IED por subsectores de la industria manufacturera se aprecia que el subsector de productos metálicos, maquinaria y equipo fue el que captó mayores flujos de IED durante el periodo 1999-2009. No obstante, dicho subsector tuvo una caída importante de flujos de inversión a partir del tercer trimestre de 2006, pasando de un monto de $\$ 4,150$ millones de dólares en 2006 a $\$ 2,927.8$ millones en 2008 (Gráfica 1). Los dos subsectores que le siguen en importancia respecto a la captación de IED son la industria de alimentos, bebidas y tabaco y las industrias metálicas básicas. En el caso del primer subsector se observa que desde el año 2004 se inicia un proceso de declinación de la inversión en este sector, mientras que las industrias metálicas básicas mostraron un comportamiento oscilatorio con grandes volúmenes de inversión en 2005 y 2007, aunque para 2008 y el primer semestre de 2009 se aprecia un contagio en la caída de los flujos de IED hacia ese subsector.

Por lo que toca específicamente a las principales ramas manufactureras receptoras de IED, de acuerdo con la Clasificación Mexicana de Actividades y Productos (CMAP), se aprecia que la industria automotriz captó el mayor volumen de inversiones entre el primer trimestre de 1999 y el tercer trimestre de 2009. Asimismo, en el Cuadro 2 se destaca que del total de las inversiones en esa rama, el 61.3\% corresponden a flujos financieros provenientes de los EUA. En segundo lugar resaltaron las inversiones realizadas en la industria básica de hierro y acero; en tercer y cuarto lugar se ubicaron las industrias de bebidas y de maquinaria, equipo y accesorios eléctricos donde se aprecia una importante participación de la IED de los EUA ( 85.97 y $60.60 \%$, respectivamente). Otras ramas manufactureras que se destacan por la captación de IED, particularmente de los EUA, son la de sustancias y productos químicos y la de ensamble de equipo electrónico de comunicaciones. 
Jorge Eduardo Mendoza Cota

Cabe destacar que al desagregar aún más las actividades manufactureras, el comportamiento de la IED por clase de manufacturas muestra que la actividad que ha tenido un mayor crecimiento es la de otras partes y accesorios para automóviles y equipo, que acumuló inversiones entre el primer trimestre de 1999 y el segundo de 2009 por un total de \$10,696.7 millones de dólares. Le siguieron en orden de importancia las industrias de otros productos no clasificados, otros productos de acero, la fabricación y ensamble de automóviles y la elaboración de refrescos y otras bebidas no alcohólicas.

Es importante señalar que la IED directa se ha canalizado principalmente hacia diversas actividades manufactureras orientadas a las exportaciones. En particular, las industrias de autopartes y la del automóvil fueron el destino fundamental de la IED, lo que está íntimamente relacionado con el establecimiento del TLCAN y el patrón de crecimiento basado en la exportación de manufacturas que se propició como resultado de dicho acuerdo. Este fenómeno, conjuntamente con el creciente volumen de exportaciones manufactureras, particularmente de las industrias relacionadas al sector automotriz, determinó un fenómeno de creciente integración de las industrias manufactureras de México con respecto al comportamiento del sector manufacturero de los EUA.

\section{LA EVOLUCIÓN DEL SECTOR MANUFACTURERO}

La evolución de las manufacturas mexicanas en la primera década del 2000 estuvo afectada por la tendencia recesiva experimentada en los EUA en 2001 y, otra vez, en 2008. Por ello, el periodo 1999-2008 mostró un crecimiento moderado del valor agregado a precios constantes en los nueve sectores manufactureros con excepción de las industrias textiles y de la madera. No obstante, al analizar el crecimiento del empleo total de trabajadores en las manufacturas se aprecia que hubo una caída generalizada en todos los subsectores manufactureros en el periodo seńalado. En ese sentido, se aprecia que el crecimiento del valor agregado per cápita de los subsectores manufactureros fue positivo para los nueve subsectores, destacándose el crecimiento de las industrias de alimentos y bebidas, de las industrias químicas y de las industrias metálicas básicas (Cuadro 3).

Este crecimiento moderado del valor de la producción de los subsectores manufactureros fue severamente disminuido por caídas importantes de la producción principalmente a partir de 2008. Además, se destaca que las ramas incluidas en el subsector de equipo de transporte fueron las que se han visto más adversamente afectadas en su ritmo de crecimiento por la recesión internacional. Se destaca que dicho subsector presentó un estancamiento en el crecimiento 
en 2007 del $0.1 \%$, seguido de una severa caída del $-13.6 \%$ en el año 2008, acumulando una caída adicional del $6.8 \%$ hasta el cuarto trimestre de 2009 . Es posible concluir que esta actividad manufacturera que incluye a las industrias productoras de automóviles y camionetas y equipo de transporte y autopartes, ha sido más afectada por la recesión internacional, particularmente la que se ha experimentado en la economía de los EuA. Lo anterior pone en evidencia que las ramas manufactureras que mostraron mayores tasas negativas de crecimiento estuvieron relacionadas con una mayor integración al comportamiento de la IED proveniente de la economía norteamericana y al efecto en la demanda de importaciones norteamericana de bienes manufacturados provenientes de México.

El siguiente subsector que entró en una severa recesión es el de la fabricación de computadoras, equipos de comunicación y electrónicos. Se destaca también que este sector venía mostrando ya los efectos del estancamiento en el crecimiento derivados del efecto de la crisis de $2001 \mathrm{y}$ del impacto generado por las exportaciones de China en el mercado norteamericano. De esta forma, las exportaciones de insumos electrónicos y componentes de computadoras también sufrieron el efecto de la reducción de la demanda de las empresas y consumidores de los EUA. Otros subsectores manufactureros que también se vieron afectados fueron la producción de muebles, de textiles y de prendas de vestir, todos ellos con tasas de crecimiento negativas en 2008 por arriba del 7\% (Cuadro 4).

Las actividades manufactureras que experimentaron la recesión económica incidieron en la tendencia negativa del sector manufacturero en su conjunto y evidenciaron la dependencia de las ramas manufactureras más dinámicas con respecto al mercado de los EuA. Lo anterior refleja, por tanto, el resultado del proceso de integración del ciclo de la producción manufacturera de los EUA con las principales ramas manufactureras exportadoras de la economía mexicana. Por lo anterior, es importante establecer un modelo de análisis econométrico que permita correlacionar los movimientos de la IED con el crecimiento del sector manufacturero, y así estar en posibilidad de obtener mayores evidencias del impacto de las inversiones externas en las manufacturas, en un contexto de integración de éstas al ciclo económico de los EUA.

\section{ASPECTOS TEÓRICOS Y METODOLÓGICOS DE LA ESTIMACIÓN DE IMPACTO DE LA IED}

La economía mexicana se caracteriza por una estrategia de exportaciones manufactureras, que en buena medida descansa en la dinámica de la industria automotriz. Algunos autores (Máttar, Moreno-Brid y W. Peres, 2002) han seńalado que 
la IED, en el marco de economías abiertas con promoción de exportaciones como la mexicana, tiende a generar mayores beneficios comparados con el caso de las economías cerradas (Bhagwati, 1978). Ello se explica por la existencia de una neutralidad de la estrategia exportadora como resultado de que las tasas de cambio efectivas, al no sesgar una tasa de cambio superior en las importaciones que en las exportaciones, promueve la IED en un contexto de menores costos, lo que conduce a resultados más eficientes. Dicha hipótesis indica que, en economías abiertas, se genera una mayor atracción de IED y un efecto de eficiencia que produce una mejor distribución de los recursos con base en ventajas comparativas.

Por otra parte, dentro de la teoría del crecimiento los modelos se han centrado en discutir el efecto del ahorro y la inversión (Harrod, 1939 y Domar, 1946), los modelos neoclásicos que enfatizan el progreso técnico (Solow, 1957) y los modelos de crecimiento endógeno que subrayan la importancia del capital humano, la investigación y el desarrollo, y las economías externas (Romer, 1986) y (Lucas, 1988).

En esta perspectiva, varios de los factores relacionados con la nueva teoría del crecimiento económico como las externalidades y la difusión tecnológica pueden vincularse también al crecimiento de la IED. Asimismo, se ha constatado que en países en desarrollo orientados a la exportación la IED tiene un impacto en la dotación de capital humano al incrementar la transferencia de habilidades del trabajo (Balasubramanyam, Salisu y Sapsford, 1996).

Con base en los enfoques teóricos mencionados, el presente estudio utiliza una función de producción de corto plazo que incluye a la IED como un insumo adicional. Se considera que la IED es una fuente directa en la generación de capital humano y tecnología por lo que permite generar externalidades y efectos de difusión. De igual modo se incluye como una variable de control a las exportaciones en la función de producción, ya que la inclusión de esta actividad económica permite generar economías de escala debido a la mejor utilización de la capacidad instalada, evita la escasez de divisas y permite acceder a mayores niveles tecnológicos (Salvatore y Hatcher, 1991). Siguiendo a De Mello (1997), y con base en el enfoque señalado anteriormente, el modelo propuesto se basa en una función de producción convencional que incluye a las exportaciones y al capital humano del sector manufacturero de México:

$Y=A F\left(L, K_{d}, E\right)=A L K_{d}^{\beta} E^{1-\alpha-\beta}$

Donde:

$\mathrm{Y}=$ crecimiento del producto manufacturero en términos reales,

$\mathrm{T}=$ factor trabajo 
$\mathrm{Kd}=$ acervo de capital

$\mathrm{A}=$ tecnología

$\alpha$ y $\beta=$ son las participaciones del factor trabajo y el factor capital en el producto manufacturero

$\mathrm{E}=$ externalidad

La externalidad puede ser expresada también en una función Cobb-Douglas de la siguiente forma:

$E=\left(L K_{d} K_{e}^{\gamma} K_{b}^{\delta}\right)^{\theta}$

En donde $\gamma$ y $\delta$ son elasticidades de sustitución entre el capital físico doméstico, el capital externo y el capital humano, y el coeficiente $\theta$ es la elasticidad de sustitución en un contexto de intertemporalidad. Dichas elasticidades son positivas, lo cual implica que adiciones de activos de inversión extranjera y de capital humano aumentarán las elasticidades del producto con respecto al trabajo y capital. Los subíndices d, e y h representan el capital doméstico, el externo y el humano. Sustituyendo 2 en 1 se obtiene:

$Y=A L^{\alpha+\theta(1-\alpha-\beta)} K_{d}^{\beta+\theta(1-\alpha-\beta)}+K_{e}^{\gamma \theta(1-\alpha-\beta)} K_{b}^{\delta \theta(1-\alpha-\beta)}$

La ecuación (3) muestra que el crecimiento de las manufacturas al nivel sectorial dependen del factor trabajo, el capital doméstico, el capital extranjero (IED) y el capital humano, y asumiendo que $\gamma$ y $\delta>0$, la IED genera externalidades positivas y es complementaria a la inversión doméstica, por lo que cualquier incremento de la IED tendrá como resultado un aumento del producto con respecto al trabajo, y capital humano. Tomando logaritmos la ecuación que sirve de base para realizar la estimación econométrica se formaliza de la siguiente manera:

$\operatorname{In}\left(\frac{Y_{t+1}}{Y}\right)=\alpha+\beta_{1} \operatorname{In}\left(L_{i t}\right)+\beta_{2} \operatorname{In}\left(K_{i t}\right)+\beta_{2} \operatorname{In}\left(K_{i t h}\right)+\beta_{4} \operatorname{In}\left(X_{i t}\right)+e_{i t}$

Donde:

$\mathrm{L}=$ total de trabajadores de la industria manufacturera por subsectores

$\mathrm{Kf}=$ inversión extranjera directa

$\mathrm{Kh}=$ proporción de empleados administrativos y técnicos respecto al total de trabajadores de la industria (proxi de capital humano)

$\mathrm{X}=\mathrm{el}$ valor de las exportaciones manufactureras sobre el producto manufacturero como aproximación del grado de apertura del sector

$\mathrm{e}_{\mathrm{it}}=$ error aleatorio de la regresión 
Jorge Eduardo Mendoza Cota

Cabe destacar que la especificación econométrica se ajustó a la disponibilidad de información estadística. Particularmente, debido a la escasez de información sobre la IED a nivel de subsectores manufactureros, los acervos de IED se estimaron con un proxi construido como la proporción de inversión extranjera respecto al producto manufacturero de cada subsector. Además, el modelo econométrico considera que la tecnología del sector manufacturero está determinada por una función que incluye al capital humano y al stock de IED existente (Borensztein, De Gregorio y Lee, 1995). En este marco analítico, las empresas de capital extranjero son un canal para transmitir el progreso tecnológico al permitir adoptar tecnologías utilizadas por las distintas variedades de inversión. Por tanto, en el modelo, la posibilidad de potenciar los efectos de la IED en el crecimiento del producto manufacturero está también en función de los niveles de capital humano empleados en el sector.

\section{Metodología de estimación}

Con el fin de estimar el efecto de la dinámica de la IED en el crecimiento del sector manufacturero de México se realizaron varias estimaciones econométricas para datos de panel anuales para el periodo 1999-2008. La estructura de los datos de panel está relacionada con la definición de grupos de acuerdo con los nueve subsectores manufactureros que presenta la Clasificación Mexicana de Actividades y Productos (CMAP). ${ }^{1}$ La ventaja de esta metodología de estimación econométrica se relaciona, entre otras cosas, con la posibilidad de contar con la mayor disponibilidad de bases de datos que se ajusten a la estructura de panel. La opción de combinar diferencias dinámicas inter e intra-individuales genera mayor información sobre datos de sección cruzada y datos de series de tiempo, al tener una mayor precisión en la inferencia estadística derivada de más grados de libertad, una menor posibilidad de problemas de multicolinealidad que en el caso de datos de sección cruzada, una mayor facilidad para determinar las pruebas de hipótesis del comportamiento económico, y finalmente, al aportar información sobre los grupos individuales y la dinámica inter-temporal de los mismos (Hsiao, 2005).

1 Productos alimenticios, bebidas y tabaco, textiles, prendas de vestir e industria del cuero, industria de la madera y productos de madera, papel, productos de papel, imprentas y editoriales, sustancias químicas, derivados del petróleo, productos de caucho y plástico, productos de minerales no metálicos, excepto derivados del petróleo y carbón, industrias metálicas básicas, productos metálicos, maquinaria y equipo y otras industrias manufactureras. 
Formalmente el modelo general de datos de panel que se estima parte de observaciones múltiples para los subsectores manufactureros que componen el total de la industria manufacturera de México y se representa de la siguiente manera:

$$
\begin{aligned}
& Y i t=\beta_{0}+\sum_{k=1}^{k} x_{k i t} \beta_{k i t}+e_{i t}, \\
& e_{i t}=\lambda_{i}+v_{i t} \\
& \mathrm{i}=1 \ldots . .9, \mathrm{t}=10
\end{aligned}
$$

Donde $i$ es el número de subsectores y $t$ es el número de periodos, $x_{k i t}$ son las variables explicativas presentadas en la ecuación (4), $k \times N \times T$ el número de coeficientes de la regresión y $e_{i t}$ es el término de error compuesto por $\lambda_{i}$ que es una constante de los individuos y $v_{i t}$ que es un error distribuido normalmente.

Con base en este modelo se pueden realizar diversas estimaciones que permiten estimar un modelo que capte las variaciones entre unidades de observación (corte transversal) y su variación temporal. De manera exploratoria se corrió un modelo de efectos fijos donde $\lambda_{i}$ se asume como constante $\left(\beta_{0}+\lambda_{i}\right)$ pero con variaciones al nivel individual.

Se estimaron las pruebas Breuch-Pagan y de Hausman. La primera prueba permite determinar la existencia de efectos fijos o individuales mediante la hipótesis nula: $H_{0}=\sigma_{\lambda}^{2}=\sigma_{\mathrm{v}}^{2}=0$, que se calcula utilizando la distribución Chi cuadrada. Por su parte, la prueba de Hausman permite identificar la interdependencia de $\lambda_{i}$ y $x_{k i t}$, mediante la hipótesis nula: $H 0=\mathrm{E}\left(\lambda_{i} \mid X_{i t}\right)=0$. La prueba estima los coeficientes con el modelo de efectos fijos y el de efectos aleatorios y su covarianza. Si la prueba no muestra correlación se cumple la hipótesis nula de no diferencia entre los coeficientes de ambos modelos.

Los resultados de la prueba Breusch-Pagan indicaron la posibilidad de que el modelo de efectos aleatorios pudiese ser más apropiado para estimar un modelo con variables de manera más eficiente. El modelo de efectos aleatorios considera que la constante entre los individuos es parte del término de error compuesto: $e_{i t}=\lambda_{i}+v_{i t}$. Este supuesto es importante para la consistencia del modelo, ya que implica la inexistencia de correlación entre los efectos no observados y las variables explicatorias.

Debido a la limitada evidencia estadística de los coeficientes obtenidos con el modelo de efectos aleatorio, considerando los resultados de aplicación de la prueba de Hausman y la evidencia de autocorrelación en las estimaciones de 
efectos fijos, reflejada en un bajo estadístico Durbin Watson, se optó por estimar un modelo de panel dinámico.

Este modelo elimina la autocorrelación de los residuos tomando la primera diferencia del modelo e incluye retrasos en la variable dependiente (instrumentos), dinamizando de esta manera el modelo. Este tipo de análisis complica la estimación del modelo porque correlaciona la variable dependiente retrasada con los términos de error, por lo que el modelo de panel debe ser calculado con estimadores IV derivados del método generalizado de momentos (GMM), que calculan retrasos para las variables explicativas en niveles. Este mecanismo permite utilizar óptimamente todas las restricciones lineares que se derivan de las especificaciones generando estimaciones asintóticamente eficientes (Arellano y Bond, 1991). Así pues, el modelo utilizado se presenta formalmente de la siguiente manera:

$Y_{i t}=Y_{i t}-1 \lambda+\sum_{k=1}^{k} X_{k i t} \beta_{k i t}+e_{i t}$

Donde $u_{i}$ desparece, la constante de los individuos $\lambda_{i}$ se integra en la ecuación y la variable dependiente retrasada y diferenciada $Y_{i t}^{i}-1$ se vuelve función del término de error retrasado $e_{i t-1}$. Este modelo permite controlar los efectos no observados y la endogeneidad de las variables explicativas.

Por tanto, el modelo asume exogeneidad estricta de las variables explicativas con respecto al término de error. A fin de verificar si la base de datos cumple con este supuesto se aplicó la prueba de Sargan, para verificar la de restricciones, cuya hipótesis nula es que los instrumentos como grupo son exógenos. Así el modelo asume que no existe correlación en el término de error, por lo que se estimó la prueba Arellano-Bond con el programa econométrico STATA.

La base de datos de panel utilizada para estimar los modelos econométricos abarca el periodo 1999 a 2008 y se construyó con información de las variables de empleo e ingreso de los trabajadores para los nueve subsectores que componen el sector manufacturero con información de la Encuesta Industrial Mensual Clasificación de acuerdo con la Clasificación Mexicana de Actividades y Productos (CMAP), publicada en el Banco de Información Económica (BIE) del Instituto Nacional de Estadística, Geografía e Informática (INEGI). Con respecto a la información sobre la inversión extranjera directa en los subsectores manufactureros, se obtuvo de la información sobre IED publicada por la Dirección de Inversión Extranjera de la Secretaría de Economía. Finalmente, las exportaciones manufactureras de acuerdo con la clasificación CMAP se obtuvieron de la balanza de productos manufacturados que publica el Banco de México (BANXICO). 
Impacto de la inversión extranjera directa en el crecimiento manufacturero en México

\section{RESULTADOS DE LA ESTIMACIÓN DE LA IED EN EL SECTOR MANUFACTURERO}

El primer modelo estimado de efectos fijos muestra que la variable de exportaciones juega un papel significativo en el crecimiento del valor agregado producido por los nueve sectores de la industria manufacturera. No obstante, el modelo mostró evidencia de autocorrelación con base en el estadístico Durbin Watson (Dw). Debido a estos resultados, se realizó la estimación de la prueba BreuschPagan para verificar la presencia de efectos aleatorios en la base de datos de panel y se estimó un modelo de panel de datos dinámico. ${ }^{2}$

El resultado de la prueba arroja un valor para el multiplicador lagrangeano con distribución chi cuadrada con un grado de libertad igual a 1.60, lo cual rechaza la hipótesis nula de no existencia de efectos aleatorios $\left(H_{0}=\sigma_{\mathrm{u}}^{2}=0\right)$; es decir que el modelo es dependiente de la varianza de los efectos individuales. Lo anterior nos conduce a estimar un modelo de panel con efectos aleatorios con base en una estimación de mínimos cuadrados generalizados (Cuadro 5).

El modelo de panel con efectos aleatorios permite estimar los parámetros que reflejan la heterogeneidad existente en las observaciones individuales de la muestra, lo que permite generar mayor inferencia estadística. Se presentan dos modelos, uno en el que la variable dependiente es el crecimiento anual del empleo de cada uno de los nueve subsectores del sector manufacturero, y otro modelo en el que la variable dependiente es el crecimiento del valor agregado en este mismo sector. Los resultados arrojan coeficientes que no son significativos estadísticamente con excepción de los coeficientes de las exportaciones y de la proporción de empleados sobre el total de trabajadores del sector manufacturero que resultaron positivos y significativos al $10 \%$ del nivel de confianza, de acuerdo con los estadísticos z correspondientes (Cuadro 6).

De esta manera, los resultados de las estimaciones de los coeficientes y la prueba de Hausman que rechazó la hipótesis nula, ${ }^{3}$ indican una correlación entre los efectos no observados y las variables explicatorias, determinaron la necesidad de profundizar el análisis de la base de datos de panel por medio de la estimación de un modelo de panel de datos dinámico. Por su parte, la hipótesis

2 Los resultados de la estimación no se presentan en el artículo debido a los problemas de autocorrelación mencionados anteriormente restan poder explicatorio al modelo.

3 La hipótesis nula de que los coeficientes del modelo de panel de efectos fijos y el de efectos aleatorios son consistentes, es decir son similares. La hipótesis alternativa implica la existencia de inconsistencia en los coeficientes de efectos aleatorios. 
nula de que los instrumentos como grupo son exógenos no se rechaza de acuerdo con la prueba de Sargan, por lo que la prueba no se debilita por el número de instrumentos (Cuadro 7).

A fin de corroborar si el modelo de panel dinámico cumplía con el supuesto básico de no correlación del término de error con las variables exógenas, se estimó la prueba de Sargan que analiza la sobre-identificación de restricciones en el modelo de panel y la prueba Arellano-Bond. Ambas pruebas no rechazaron las hipótesis nulas correspondientes, por lo que las estimaciones se ajustan a los supuestos y condiciones del modelo de panel dinámico.

Los resultados de las estimaciones del modelo de panel dinámico muestran que los coeficientes de los ingresos laborales promedio fueron negativos y significativos al $1 \%$ del nivel de confianza, lo cual se conforma con el planteamiento esperado en el modelo teórico adoptado en este estudio. Asimismo, el coeficiente de las exportaciones manufactureras resultó positivo y estadísticamente significativo al 5\% de confianza, por lo que existe evidencia de que las exportaciones manufactureras se han convertido en un factor de crecimiento para el sector. Por otra parte, el coeficiente de la IED mostró un valor positivo muy reducido, pero no fue estadísticamente significativo, lo cual no permite concluir una influencia directa de la IED como un factor en el crecimiento del empleo en el sector manufacturero de México (Cuadro 8). Este resultado concuerda con lo señalado por De Mello (1999), quien señala que en la práctica es difícil estimar una relación positiva del efecto de IED en el crecimiento de los países receptores, tanto en estimaciones econométricas de series de tiempo como en los modelos de panel. Lo anterior, debido a las especificidades de las regiones o de los países, tales como las distorsiones en los retornos sociales y privados a la inversión, la aplicación de tecnologías menos productivas en países de menor desarrollo, etcétera. En el caso de la economía mexicana, al parecer los estados con menor desarrollo económico podrían estar afectando negativamente el impacto positivo de la IED en el crecimiento económico regional. Adicionalmente, la falta de impacto de IED en el crecimiento económico puede estar asociada a la IED que se orienta hacia las fusiones o adquisiciones de empresas existentes en México, lo que genera la ventaja única de ingresar divisas y, finalmente, a que la IED ha limitado la posibilidad del gobierno de promover una política de crecimiento industrial exitosa (Pacheco, 2005).

Finalmente, el coeficiente del crecimiento de empleados sobre el total de trabajadores del sector manufacturero exhibió un coeficiente positivo y estadísticamente significativo al $1 \%$ de nivel de confianza, lo que indica que en el modelo que incluye IED, el incremento de trabajadores relativamente más calificados ha permitido el crecimiento del empleo del sector manufacturero. 
Por lo que puede concluirse que el efecto de la IED en el crecimiento de las manufacturas en México se relaciona con el efecto positivo de las exportaciones manufactureras y la expansión de la calificación de la fuerza de trabajo en ese sector productivo. Este resultado concuerda con los hallazgos obtenidos en el trabajo de Borensztein, De Gregorio y Wha (1995), que indican que el efecto positivo de la IED en el crecimiento económico solamente se obtiene dependiendo de la dotación de capital humano de los países receptores. Concuerda con los resultados de Nunnenkamp, Alatorre y Waldkirch (2007) que encuentran también un mayor efecto de la IED en el crecimiento del empleo de trabajadores calificados.

\section{CONCLUSIONES}

En la literatura sobre la IED se ha argumentado que ésta ha sido decisiva en el crecimiento de las economías receptoras, argumentándose aspectos relacionados con el acervo de capital existente en la economía, una mayor productividad del trabajo en las empresas multinacionales, la generación de economías externas en dicho sector industrial, en especial en regiones donde existe una alta concentración de IED, y la difusión de la tecnología. Se ha argumentado que el efecto de la IED puede ser también no concluyente e incluso puede ser negativo dependiendo de las condiciones particulares de los países receptores.

Debido a la importancia que reviste el análisis del impacto de la IED en el crecimiento económico de los países altamente receptores de esta inversión como es el caso de la economía mexicana, el presente estudio se orientó a realizar una estimación del efecto de la IED en el crecimiento del sector manufacturero de México, debido a su importancia como eje del modelo orientado a las exportaciones, seguido en la economía mexicana desde que se implementó la liberalización económica.

El comportamiento de la IED canalizada a los subsectores manufactureros, muestra que ésta experimentó un gran dinamismo durante la década de los noventa. No obstante, en la primera década del 2000 se inicia una etapa de caída, estancamiento y fluctuaciones de la IED en el sector manufacturero. A partir de esa fecha, el sector manufacturero mostró un comportamiento oscilatorio en el crecimiento promedio en el valor agregado producido, lo que fue afectado por fluctuaciones recesivas al inicio y al final de esa década. Particularmente, esta característica se aprecia claramente en las manufacturas de transporte, computación y textiles, estando estas ramas vinculadas significativamente a los movimientos de la IED y las exportaciones al mercado estadounidense. 
Para realizar el análisis de la relación entre la IED y el crecimiento manufacturero, se utilizaron modelos de panel que permiten estimar el impacto de los flujos de IED y el crecimiento de los nueve subsectores que componen las manufacturas de México, de acuerdo con la cMap. Lo anterior con la finalidad de utilizar la parte longitudinal de tiempo de la series al rezagar la variable dependiente. Debido a problemas de autocorrelación serial exhibido en los resultados del modelo de efectos aleatorios, y al objetivo de determinar si la dinámica de crecimiento del sector manufacturero está vinculada a los efectos retrasados de la IED, se estimó un modelo de panel dinámico. Con base en los modelos estimados para la base de datos de panel del sector manufacturero se aprecian los siguientes resultados.

Las exportaciones manufactureras, que reflejan el grado de apertura del sector, muestran un signo positivo y estadísticamente significativo en las estimaciones de efectos aleatorios y de panel dinámico. Lo anterior corrobora que el efecto de la apertura ha sido factor de dinamismo del crecimiento del sector manufacturero, al generar mercados y, en conjunción con la IED, promover el dinamismo de algunas actividades manufactureras para la exportación.

Las estimaciones mostraron un efecto positivo muy reducido de la IED y de poca significancia estadística en el crecimiento del empleo del sector manufacturero, por lo que este resultado no es concluyente en cuanto al papel que directamente juega la IED en el crecimiento del sector manufacturero. Por otra parte, el modelo que estima el impacto de la IED en el crecimiento del valor agregado de la industria manufacturera (ieva) mostró un coeficiente negativo y estadísticamente no significativo. Por ello, los resultados no confirman estimaciones previas donde existe un efecto marginalmente positivo de la IED en el crecimiento. Lo anterior probablemente debido a la heterogeneidad del sector manufacturero, y al periodo analizado que recoge las dos etapas recesivas de 2001 y de 2008-2009 experimentadas en la economía mexicana.

No obstante, las estimaciones mostraron un coeficiente positivo y significativo de la variable de la proporción de empleados administrativos y técnicos sobre el total de trabajadores, lo que permite asumir que la mayor capacitación del trabajo ha determinado un crecimiento del sector manufacturero en su conjunto. Lo anterior apoya la perspectiva de trabajos previos que encontraron que el crecimiento del capital humano, que se expande mediante los flujos de IED. Dicho resultado podría considerarse como una evidencia indirecta de los efectos positivos en el crecimiento de la IED.

De esta manera, los resultados del modelo econométrico apoyan parcialmente la hipótesis de la existencia de efectos positivos de la IED en el crecimiento de la industria manufacturera al nivel sub-sectorial. Este hecho contrasta los hallazgos de trabajos previos sobre el impacto de la IED en el crecimiento del total de las 
actividades económicas de los países receptores, que han encontrado efectos positivos. Lo anterior puede derivarse, en primer término, de la diferencia en las bases de datos utilizados, pues la mayoría de los trabajos que han encontrado efectos positivos se realizan para muestras al nivel de países. Dicha metodología no incorpora de manera más amplia las particularidades de las economías receptoras, por lo que limita la capacidad de retomar las especificidades del conjunto de las economías y su respuesta ante los flujos de inversión extranjera.

Por otra parte, cabe recalcar que el análisis de este estudio es de carácter sectorial. En esta perspectiva, debe destacarse que el análisis no toma en consideración el efecto que tiene la IED en el sector servicios, que al generar mayor valor agregado comparativamente con el sector manufacturero, particularmente en economías en desarrollo como México, podría estar dejando de considerar el impacto de este sector en el crecimiento del conjunto de actividades económicas.

Finalmente, es importante destacar que la información sobre industria manufacturera incorpora elementos de heterogeneidad geográfica tanto al nivel de estados, como al nivel de los nueve sub-sectores de la industria manufacturera. Por una parte se encuentran actividades manufactureras con una gran recepción de IED y producción comparativamente intensiva en capital y para la exportación, como es el caso de la industria automotriz y de autopartes y, por otro, se tienen industrias como las de alimentos, que producen predominantemente para el mercado local y que presentan menor intensidad de capital. Es posible que los resultados econométricos capturen el efecto de la IED en la demanda de trabajadores más calificados, demostrando su efecto de difusión tecnológica, mientras que la heterogeneidad no permita capturar claramente el efecto de la IED en el crecimiento del empleo y del valor agregado del sector manufacturero.

\section{BIBLIOGRAFÍA}

Aitken, B., G. Hanson and A. Harrison, "Spillovers, Foreign Investment and Export Behavior", Journal of International Economics, vol. 43, num. 1-2, 1997, pp. 103-32.

Arellano, M. and S. Bond, "Some Tests of Specification for Panel Data: Monte Carlo Evidence and an Application to Employment Equations", The Review of Economic Studies, vol. 58, num. 2, 1991, pp. 277-297.

Balasubramanyam, V. N., M. Salisu and D. Sapsford, "Foreign Direct Investment and Growth in EP and Is Countries", Economic Journal, num. 106, 1996, pp. 96-105. 
Jorge Eduardo Mendoza Cota

Bhagwati, J. N., "Anatomy and Consequences of Exchange Control Regimes", National Bureau of Economic Research, vol. I, Studies in International Economic Relations, num. I0, New York, 1978.

Blomström, M., y Persson, "Foreign Investment and Spillover Efficiency in an Underdeveloped Economy: Evidence from the Mexican Manufacturing Industry", World Development, num. 11, 1983, pp. 493-501.

Blomström, M., y A. Kokko, "Regional Integration and Foreign Direct Investment”, NBER Working Paper, num. 6019, 1997.

Borensztein E., J. de Gregorio y J., Wha Lee, "How Does Foreign Direct Investment Affect Economic Growth", National Bureau of Economic Research, 1995, Working Paper 5057.

"How Does Foreign Investment Affect Economic growth?", NBER Working Paper Series, Working Paper, num 5057, 1995.

De Gregorio José, "The Role of Foreign Direct Investment and Natural Resources in Economic Development", Banco Central de Chile, Documento de trabajo, núm. 196, 2003.

De Mello, Jr., Luiz R., "Foreign Direct Investment in Developing Countries and Growth: A Selective Survey", The Journal of Development Studies, vol. 40, num. 3, 1997, pp. 1-34.

"Foreign Investment-led Growth: Evidence From Times Series and Panel Data", Oxford Economic Papers, num. 51, 1999, pp. 133-151.

Domar, Evsey, "Capital Expansion, Rate of Growth, and Employment", Econometrica, vol. 14, num. 2, 1946, pp. 137-147.

Dussel, P. E., L. M. Galindo, E. Loría y M. Mortimore, Inversión extranjera directa en México: desempeño y potencial, México, Siglo xxi editores, 2007.

Hausman, J.A., "Specification Tests in Econometrics", Econometrica, vol. 46, num. 6, 1978, 1251-1271.

Hausman, J. A. and W. E. Taylor, "Panel Data and Unobservable Individual Effects”, Econometrica, vol, 49, num. 6, 1981, pp. 509-533.

Harrod, Roy, "An Essay in Dynamic Theory," Economic Journal 49, 1939, pp. 14-33.

Herzer, D., S. Klasen y F. Nowak-Lehmann, "In Search of FDI-led Growth in Developing Countries", Instituto Ibero-Americano de Estudios Económicos, University of Goettingen, (http://ideas.repec.org/p/got/iaidps/150.html), 2006.

Hsiao, Cheng, "Why Panel Data?", Institute of Economic Policy Research, University of Southern California, Working Paper 05.33, 2005. 
, "State Characteristics and the Locational Choice of Foreign Direct Investment: Evidence from Regional FDI in Mexico 1989-2006", Growth and Change, vol. 39, num 3, 2008, pp. 389-413.

Jordaan Jacob, "Determinants of FDI-Induced Externalities: New Empirical Evidence for Mexican Manufacturing Industries", World Development, vol. 33, num.12, 2005, pp. 2103-18.

Kokko, A., "Technology, Market Characteristics, and Spillovers", Journal of Development Economics, vol. 43, num. 2, 1994, pp. 279-293.

Love J. H. y F. Lage-Hidalgo, "Analysing the Determinants of us Direct Investment in Mexico", Applied Economics, vol. 32, num. 10, 2000, pp. 1259-1267.

Lucas Robert, "On the Mechanics of Economic Development", Journal of Monetary Policy, vol. 22, num. 88, 1988, pp. 3-39.

Máttar J., J. C. Moreno-Brid y W. Peres, "Foreign Investment in Mexico After Economic Reform”, Cepal, México, Serie Estudios y Perspectivas, 2002, num. 10.

Nunnenkamp P., J. E. Alatorre y A. Waldkirch, "FDI in Mexico: An Empirical Assessment of Employment Effects", Kiel Institute for the World Economy, Working Paper num. 1328, 2007.

Pacheco-López, P., "Foreign Direct Investment, Exports and Imports in Mexico", The World Economy, vol. 28, num. 8, 2005, pp. 1157-1172.

Ramirez, M.D., "Foreign Direct Investment in Mexico: A Cointegration Analysis", The Journal of Development Studies, vol. 37, num. 1, 2000, pp. 138-162.

, "Foreign Direct Investment in Mexico During the 1990s: An Empirical Assessment", Eastern Economic Journal, vol. 28, num. 2, 2002, pp. 409-423.

Romer, P. M., "Increasing Returns and Long-run Growth", Journal of Political Economy, vol. 94, num. 5, 1986, pp. 1002-1037.

Romer, P. M., "Growth Based on Increasing Returns due to Specialization", American Economic Review (Papers and Proceedings), vol. 77, 1987, pp. 56-62 .

Salvatore, D. and T. Hatcher, "Inward oriented and Outward Oriented Trade Strategies", Journal of Development Studies, vol. 27, num. 3, 1991, pp. 7-25.

Solow, Robert, "Technical Change and the Aggregate Production Function", Review of Economics and Statistics, vol. 39, num. 3, 1957, pp. 312-320. 
Cuadro $]$

Evolución de la Inversión Extraniera Directa en el Sector Manufacturero de México, 1994-2008

\begin{tabular}{lcc}
\hline Periodo & Inversión Extranjera Total & $\begin{array}{c}\text { Inversión Extraniera Directa en el sector } \\
\text { manufacturero como porcentaje } \\
\text { del total de la IED }\end{array}$ \\
\hline 1994 & $\$ 10,646.9$ & $58.16 \%$ \\
1995 & $\$ 8,374.6$ & $58.06 \%$ \\
1996 & $\$ 7,847.9$ & $61.41 \%$ \\
1997 & $\$ 12,145.6$ & $60.03 \%$ \\
1998 & $\$ 8,373.5$ & $59.83 \%$ \\
1999 & $\$ 13,858.6$ & $66.11 \%$ \\
2000 & $\$ 18,019.6$ & $55.32 \%$ \\
2001 & $\$ 29,817.7$ & $19.82 \%$ \\
2002 & $\$ 23,728.9$ & $37.07 \%$ \\
2003 & $\$ 16,521.6$ & $46.91 \%$ \\
2004 & $\$ 23,681.1$ & $55.77 \%$ \\
2005 & $\$ 21,976.8$ & $50.21 \%$ \\
2006 & $\$ 19,428.0$ & $50.85 \%$ \\
2007 & $\$ 27,528.1$ & $44.28 \%$ \\
2008 & $\$ 22,481.2$ & $29.04 \%$ \\
\hline
\end{tabular}

Fuente: Estimaciones propias con datos elaborados por la Secretaría de Economía. Dirección General de Inversión Extranjera publicados en el Banco de Información Económica de INEGl.

Cuadro 2

Principales ramas manufactureras receptoras de IED, 1999/01-2009/03

Millones de dólares

\begin{tabular}{|c|c|c|}
\hline Rama & $\begin{array}{c}\text { IED } \\
\text { acumulada }\end{array}$ & $\begin{array}{l}\text { IED de EUA entre } \\
\text { el total de IED }\end{array}$ \\
\hline 384100 Industria automotriz & $\$ 17,549$ & $61.30 \%$ \\
\hline 371000 Industria básica del hierro y del acero & $\$ 7,973$ & $8.29 \%$ \\
\hline 313000 Industria de las bebidas & $\$ 7,586$ & $85.97 \%$ \\
\hline $\begin{array}{l}383100 \text { Fabricación y/o ensamble de maquinaria, equipo y accesorios } \\
\text { eléctricos. Incluso para la generación de energía eléctrica. }\end{array}$ & $\$ 6,914$ & $60.60 \%$ \\
\hline 352200 Fabricación de otras sustancias y productos químicos. & $\$ 6,651$ & $75.55 \%$ \\
\hline 390000 Otras industrias manufactureras. & $\$ 6,380$ & $88.41 \%$ \\
\hline $\begin{array}{l}383200 \text { Fabricación y/o ensamble de equipo electrónico de radio, televisión, } \\
\text { comunicaciones y de uso médico. }\end{array}$ & $\$ 6,025$ & $69.54 \%$ \\
\hline 312100 Elaboración de otros productos alimenticios para el consumo humano & $\$ 4,879$ & $25.47 \%$ \\
\hline $\begin{array}{l}382200 \text { Fabricación, reparación y/o ensamble de maquinaria y equipo para } \\
\text { usos generales, con } 0 \text { sin motor eléctrico integrado. Incluye armamento. }\end{array}$ & $\$ 3,141$ & $77.34 \%$ \\
\hline $\begin{array}{l}382300 \text { Fabricación y/o ensamble de máquinas de oficina, cálculo y } \\
\text { procesamiento informático. }\end{array}$ & $\$ 3,052$ & $28.28 \%$ \\
\hline
\end{tabular}


Impacto de la inversión extranjera directa en el crecimiento manufacturero en México

Fuente: Secretaría de Economía. Dirección General de Inversión Extraniera.

Cuadro 3

Tasas de crecimiento promedio anual del valor agregado, empleo y producto per cápita de los subsectores manufactureros (1999-2008)

\begin{tabular}{|c|c|c|c|c|c|c|c|c|c|}
\hline & 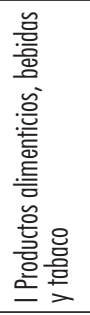 & 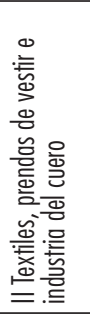 & 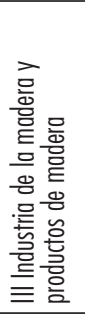 & 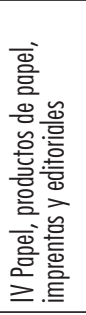 & 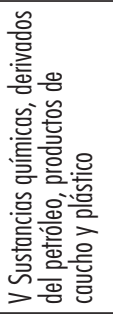 & 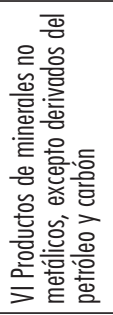 & 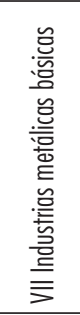 & 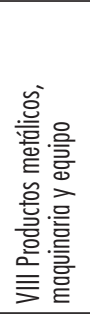 & 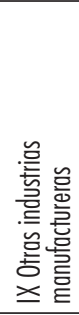 \\
\hline $\begin{array}{l}\text { Valor } \\
\text { agregado } \\
\text { (1) }\end{array}$ & $2.22 \%$ & $-1.73 \%$ & $-2.95 \%$ & $0.24 \%$ & $2.20 \%$ & $0.98 \%$ & $7.20 \%$ & $0.23 \%$ & $0.97 \%$ \\
\hline $\begin{array}{l}\text { Personal } \\
\text { ocupado } \\
\text { total }\end{array}$ & $-0.34 \%$ & $-5.56 \%$ & $-3.73 \%$ & $-0.75 \%$ & $-1.93 \%$ & $-0.85 \%$ & $-0.53 \%$ & $-3.04 \%$ & $-2.65 \%$ \\
\hline $\begin{array}{l}\text { Valor } \\
\text { agregado } \\
\text { per cápita }\end{array}$ & $2.51 \%$ & $1.99 \%$ & $0.08 \%$ & $1.03 \%$ & $2.86 \%$ & $1.14 \%$ & $5.86 \%$ & $1.17 \%$ & $0.80 \%$ \\
\hline
\end{tabular}

Fuente: Elaboración propia con datos de la Encuesta Industrial Mensual de INEGI.

' Deflactado con el Índice Nacional de Precios al Consumidor, base junio de 2002.

Cuadro 4

Los cinco subsectores de la industria manufacturera mexicana con mayores caídas en sus tasas de crecimiento anual entre 2008 y 2009

\begin{tabular}{|c|c|c|c|c|c|c|}
\hline Periodo & 2005 & 2006 & 2007 & 2008 & 2009 & $\begin{array}{l}\text { Acumulado } \\
\text { 2008-20091 }\end{array}$ \\
\hline $\begin{array}{l}\text { Fabricación de equipo de } \\
\text { transporte }\end{array}$ & $5.64 \%$ & $4.38 \%$ & $0.09 \%$ & $-13.64 \%$ & $-6.77 \%$ & $-20.41 \%$ \\
\hline $\begin{array}{l}\text { Fabricación de equipo de } \\
\text { computación, comunicación, } \\
\text { medición y de otros equipos, } \\
\text { componentes y accesorios } \\
\text { electrónicos }\end{array}$ & $-0.54 \%$ & $-0.73 \%$ & $4.87 \%$ & $-7.84 \%$ & $-4.67 \%$ & $-12.52 \%$ \\
\hline $\begin{array}{l}\text { Fabricación de muebles y } \\
\text { productos relacionados }\end{array}$ & $3.78 \%$ & $1.50 \%$ & $-2.81 \%$ & $-8.90 \%$ & $-2.65 \%$ & $-11.55 \%$ \\
\hline $\begin{array}{l}\text { Confección de productos textiles, } \\
\text { excepto prendas de vestir }\end{array}$ & $0.10 \%$ & $1.43 \%$ & $-4.20 \%$ & $-8.80 \%$ & $-0.99 \%$ & $-9.79 \%$ \\
\hline Fabricación de insumos textiles & $-1.49 \%$ & $-1.74 \%$ & $-6.85 \%$ & $-7.92 \%$ & $-1.25 \%$ & $-9.17 \%$ \\
\hline Fabricación de prendas de vestir & $-3.92 \%$ & $0.55 \%$ & $-1.57 \%$ & $-7.22 \%$ & $-1.95 \%$ & $-9.16 \%$ \\
\hline
\end{tabular}

Fuente: Estimaciones propias con datos del INEGI. Encuesta Industrial Mensual (EIM).

1 Hasta abril de 2009. 
Jorge Eduardo Mendoza Cota

Cuadro 5

Prueba del multiplicador Lagrangeano para efectos aleatorios de Breusch y Pagan

\begin{tabular}{llc}
\hline & \multicolumn{1}{c}{ Var } & sd $=$ sqrt (Var) \\
Tee & 0.0013 & 0.0371 \\
$\mathrm{E}$ & 0.001 & 0.034 \\
& Prob $>$ chi2 $=$ & 0.2058 \\
Prueba Var $(u)=0$ & chi2 $(1)=$ & \\
& Prob $>$ chi2 $=$ & 0.86 \\
& 0.007 & 0.353 \\
Tcva & 0.006 & 0.0840 \\
E & Prob $>$ chi2 $=$ & 0.081 \\
& & 0.2058 \\
Prueba Var $(u)=0$ & chi2 $(1)=$ & 1.60 \\
& Prob $>$ chi2 $=$ & 0.206 \\
&
\end{tabular}

La prueba rechaza la HO de que $\sigma_{v}^{2}=0$.

Cuadro 6

Análisis de panel: efectos aleatorios

Variable dependiente: crecimiento del va del manufacturero (1999-2008)

90 observaciones

\begin{tabular}{|c|c|c|}
\hline VD & tce & tva \\
\hline cons & -0.035 & -0.156 \\
\hline z estadístico & $(-0.671)$ & $(-1.363)$ \\
\hline rp & 0.003 & 0.088 \\
\hline Z estadístico & $(2.23)^{\star \star}$ & $(0.27)$ \\
\hline ieva & 0.003 & -0.006 \\
\hline z estadístico & $(0.90)$ & $(-0.852)$ \\
\hline$x$ & 0.007 & 0.002 \\
\hline z estadístico & $1.83^{\star \star}$ & $(0.22)$ \\
\hline kha & 0.006 & -0.007 \\
\hline Z estadístico & $1.79^{\star \star}$ & $(-0.65)$ \\
\hline R-sq & 0.735 & 0.195 \\
\hline Wald chi2(4) & 11.75 & 8.87 \\
\hline Prob >chi2 & 0.002 & 0.064 \\
\hline
\end{tabular}


Los asteriscos ${ }^{\star \star} y^{\star * *}$ indican significancia estadística al $5 \%$ y $1 \%$.

Cuadro 7

Prueba de correlación del componente de error en el panel de efectos aleatorios de Hausman

Variable dependiente: tce

Prueba: Ho: la diferencia en los coeficientes no es sistemática.

$\operatorname{chi} 2(4)=16.15$

Prob>chi2 $=0.0883$

Variable dependiente: tva

Prueba: Ho: la diferencia en los coeficientes no es sistemática.

chi2 $(4)=16.55$

Prob>chi2 $=0.0617$

Los resultados rechazan la $\mathrm{HO}=$ de igualdad de los coeficientes de efectos fijos, mostrando inconsistencia del modelo de efectos aleatorios al $5 \%$ de confianza.

Cuadro 8

Análisis de panel dinámico, estimación Arellano-Bond (one step incluyendo variables dictómicas)

\begin{tabular}{|c|c|c|}
\hline \multicolumn{3}{|c|}{ Variable dependiente: crecimiento del empleo y del valor aqreaado manufacturero (1999-2008 } \\
\hline \multicolumn{3}{|l|}{72 observaciones } \\
\hline VD & tce & $t v a$ \\
\hline & 0.1032 & -0.162 \\
\hline z estadístico & $(0.84)$ & $(-1.38)$ \\
\hline $\mathrm{Rp}$ & $(-0.532)$ & 0.1552 \\
\hline z estadístico & $(-3.86)^{\star \star \star}$ & (0.35) \\
\hline ied & 0.001 & -0.009 \\
\hline z estadístico & (1.04) & $(-3.09)$ \\
\hline$x$ & 0.052 & -0.1994 \\
\hline z estadístico & $(1.85)^{\star \star}$ & $(-2.55)^{\star \star \star}$ \\
\hline kha & 0.283 & 0.381 \\
\hline z estadístico & $(2.92)^{\star \star \star}$ & (1.13) \\
\hline Constante & 0.014 & -0.012 \\
\hline z estadístico & $(3.60)^{\star \star \star}$ & $(-1.13)$ \\
\hline \multicolumn{3}{|c|}{ Sargan test de sobre-identificación de restricciones } \\
\hline Chi2(35) & 89.21 & 61.26 \\
\hline Prob >chi2 & 0 & 0 \\
\hline
\end{tabular}

Arellano-Bond test de que el promedio de las autocovarianzas en los residuos de orden 1 son 0 ( $\mathrm{HO}$ : sin autocorrelación):

\begin{tabular}{lll}
\hline Z estadístico & -1.75 & -4.47
\end{tabular}

tce= tasa de crecimiento anual del empleo manufacturero, tva = tasa de crecimiento anual del valor agregado manufacturero, tcp =tasa de crecimiento anual de la productividad manufacturera, ieva = inversión extranjera directa sobre valor agregado, $x=$ exportaciones manufactureras, $r p=$ remuneraciones promedio anual, ied = inversión extranjera directa anual en el sector manufacturero, kha = empleados sobre el total de trabajadores en la industria manufacturera Los asteriscos ${ }^{\star \star} y^{\star \star \star}$ indican significancia estadística al $5 \%$ y $1 \%$. 
Jorge Eduardo Mendoza Cota

Análisis de panel dinámico, estimación Arellano-Bond (one step incluyendo variables dictómicas)

\begin{tabular}{|c|c|c|c|c|}
\hline \multicolumn{5}{|c|}{ Variable dependiente: crecimiento del empleo y del valor agregado manufacturero (1999-2008) } \\
\hline \multicolumn{5}{|c|}{72 observaciones } \\
\hline VD & tce & tva & tce & tva \\
\hline & 0.1032 & -0.162 & 0.445 & -0.203 \\
\hline z estadístico & -0.84 & $(-1.38)$ & $(2.903)^{\star \star \star}$ & $(-1.194)$ \\
\hline $\operatorname{Rp}$ & $(-0.532)$ & -0.155 & -1.185 & -0.131 \\
\hline z estadístico & $(-3.86)^{\star \star \star}$ & -0.35 & $(-2.500)^{\star \star}$ & $(-1.924)^{\star}$ \\
\hline ieva & & & $(-0.001)$ & -0.0001 \\
\hline z estadístico & & & -1.531 & $(-0.929)$ \\
\hline ied & 0.001 & -0.009 & & \\
\hline z estadístico & $(1.04)$ & $(-3.09)$ & & \\
\hline$x$ & -0.052 & -0.1994 & -0.21 & -0.223 \\
\hline z estadístico & $(1.85)^{\star \star}$ & $(-2.55)^{\star \star \star}$ & $(-2.542)^{\star \star}$ & $(-2.894)^{\star \star \star}$ \\
\hline kha & 0.283 & 0.381 & 0.608 & 0.709 \\
\hline z estadístico & $(2.92)^{\star \star \star}$ & -1.13 & $(2.451)^{\star \star}$ & $(2.226)^{\star \star \star}$ \\
\hline Constante & 0.014 & -0.012 & 0.007 & 0.126 \\
\hline z estadístico & $(3.60)^{\star \star \star}$ & $(-1.13)$ & $(2.779)^{\star \star \star}$ & $(3.11)^{\star \star \star}$ \\
\hline \multicolumn{5}{|c|}{ Sargan test de sobre-identificación de restricciones } \\
\hline Chi cuadrada(35) & 89.21 & 61.26 & 43.688 & 37.864 \\
\hline Prob >chi2 & 0 & 0 & 0.008 & 0.003 \\
\hline \multicolumn{5}{|l|}{ Prueba de Wald } \\
\hline Wald chi(35) & $7.05598[0.2165]$ & $6.925[0.226]$ & $23.688(0.008)$ & $19.523(0.001)$ \\
\hline $\begin{array}{l}\text { tce = tasa de crecimier } \\
\text { ro, tcp =tasa de crecin } \\
\text { do, } x=\text { exportaciones } \\
\text { sector manufacturero, } \\
\text { Los asteriscos }{ }^{*},\end{array}$ & $\begin{array}{l}\text { anual del empleo man } \\
\text { nto anual de la producti } \\
\text { anufactureras, rp = rem } \\
\text { la = empleados sobre e }\end{array}$ & $\begin{array}{l}\text { facturero, tva = tasa } \\
\text { idad manufacturera, } \\
\text { Ineraciones promedio } \\
\text { total de trabajadores }\end{array}$ & $\begin{array}{l}\text { ecimiento anual del } \\
=\text { inversión extranje } \\
\text { al, ied = inversión e } \\
\text { a industria manufact }\end{array}$ & $\begin{array}{l}\text { agregado manufacture- } \\
\text { recta sobre valor agrega- } \\
\text { jera directa anual en el }\end{array}$ \\
\hline
\end{tabular}


Gráfica 1

Evolución de IED en la industria manufacturera de México por subsectores (1999-2009-2)

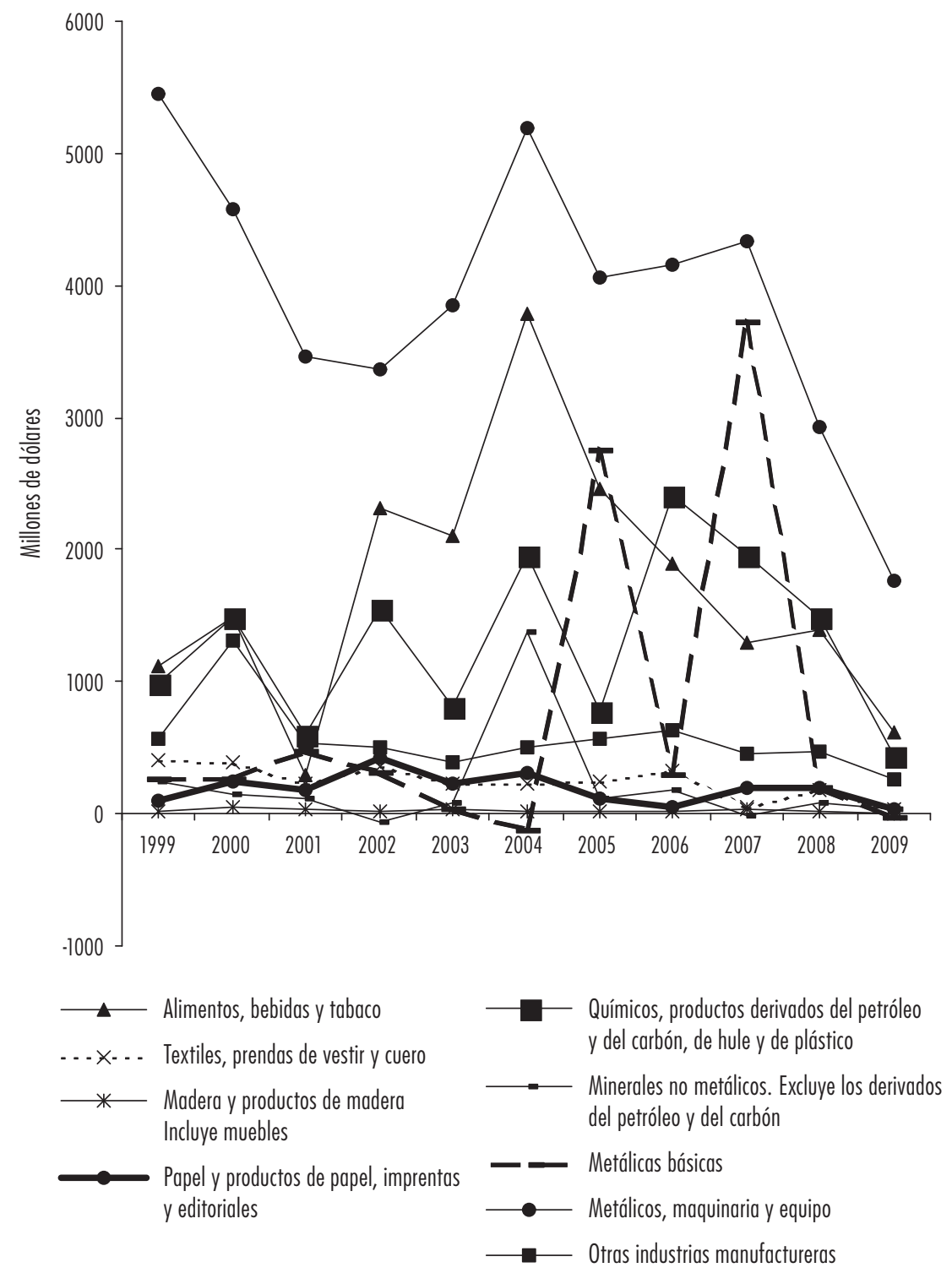

Fuente: Secretaría de Economía. Dirección General de Inversión Extranjera. 
